\title{
Oxidative Stress Assessment in Different Ethnic Groups of Girls with Exogenous Constitutional Obesity Complicated by Non-Alcoholic Fatty Liver Disease
}

\author{
Marina A. Darenskaya, $\mathrm{PhD}, \mathrm{ScD}^{1^{*}}$; Lyubov V. Rychkova, $\mathrm{PhD}, \mathrm{ScD}^{1}$; Larisa A. Zhdanova, \\ $\mathrm{PhD}^{2}$; Lyudmila A. Grebenkina, $\mathrm{PhD}, \mathrm{ScD}^{1}$; Oksana A. Gavrilova, $\mathrm{MD}^{1}$; Elena V. Osipova, \\ $\mathrm{PhD}^{1,3}$; Natalya V. Semenova, $\mathrm{PhD}^{1}$; Sergey I. Kolesnikov, Academician of the RAS ${ }^{1,4,5}$; \\ Lyubov I. Kolesnikova, Academician of the RAS ${ }^{1,3}$
}

\author{
${ }^{1}$ Scientific Centre for Family Health and Human Reproduction Problems, Irkutsk, Russia \\ ${ }^{2}$ Children's Republican Clinical Hospital of the Republic of Buryatia, Ulan-Ude, Russia \\ ${ }^{3}$ Irkutsk State University, Irkutsk, Russia \\ ${ }^{4}$ M. V. Lomonosov Moscow State University, Moscow, Russia \\ ${ }^{5}$ Moscow Region State University, Moscow, Russia
}

\begin{abstract}
The purpose of this study was to assess the lipid peroxidation (LPO) and antioxidative defense (AOD) indicators in different ethnic groups of adolescent girls with exogenous constitutional obesity (ECO) and non-alcoholic fatty liver disease (NAFLD).

Materials and Methods: A total of 20 Mongoloid teenage girls and 20 Caucasian teenage girls with NAFLD (Clinical group 1 and Clinical group 2, respectively) on the background of ECO of the first degree were examined. For comparison, data of 28 healthy Mongoloid teenage girls (Control group 1) and 20 Caucasian teenage girls (Control group 2) were used. The plasma level of antioxidant parameters (total antioxidant activity [TTA], SOD activity, $\alpha$-tocopherol and retinol) and primary/secondary products of LPO (conjugated dienes [CD], ketodienes and conjugated trienes [KD-CT], and thiobarbituric acid reactive substances [TBARS]) were determined using spectrophotometric and fluorometric methods.

Results: Evaluation of the activity of LPO reactions in Clinical group 1 indicated an increase in the content of CD, KD$\mathrm{CT}$, and TBARS relative to Control group 1. In Clinical group 2, we found an increased CD content relative to Control group 2. Interethnic differences resulted in elevated levels of KD-CT in Clinical group 1 compared to Clinical group 2.In the parameters of the AOD system, we observed reduced levels of TAA, retinol and glutathione-S-transferase (G-S-T) activity in Clinical group 2 compared to Control group 2. In Clinical group 1, we observed reduced levels of $\alpha$-tocopherol, retinol and G-S-T activity compared to Control group 1. There were no differences in the parameters of the AOD system between Clinical group 1 and Clinical group 2.

Conclusion: The results obtained indicate high activity of LPO processes and severe deficiency of antioxidant factors in Mongoloid girls with ECO and NAFLD in comparison with Caucasian girls with obesity and NAFLD and the control group. The obtained results allow us to recommend administration of antioxidant drugs in addition to basic therapy in comprehensive treatment of patients with NAFLD. (International Journal of Biomedicine. 2019;9(3):223-227.)
\end{abstract}

Key Words: adolescent $\bullet$ oxidative stress $\bullet$ obesity $\bullet$ non-alcoholic fatty liver disease

\section{Abbreviations}

AOD, antioxidative defense; CD, conjugated dienes; ECO, exogenous constitutional obesity; GR, glutathione reductase; GPO, glutathione peroxidase; G-S-T, glutathione-S-transferase; GSH, reduced glutathione; GSSG, oxidized glutathione; KD-CT, ketodienes and conjugated trienes; LPO, lipid peroxidation; NAFLD, non-alcoholic fatty liver disease; OS, oxidative stress; SOD, superoxide dismutase; TAA, total antioxidant activity; TBARS, thiobarbituric acid reactive substances. 


\section{Introduction}

Currently, NAFLD is an increasingly common form of chronic liver disease ${ }^{(1-3)}$ in both adults and children. This pathology in people who drink little or no alcohol is characterized by the presence of lipid deposits in hepatocytes. ${ }^{(4)}$ The significance of NAFLD lesions ranges from hepatic steatosis and non-alcoholic steatohepatitis, to progressing fibrosis and cirrhosis. $^{(3,4)}$ The NAFLD growth in children and adolescents is considered to have a close correlation with the prevalence of obesity. ${ }^{(5)}$ Although NAFLD has become more common, its underlying mechanisms are still unclear; hereupon, effective treatment for the disease has not been yet developed. It has been suggested that multiple parallel factors, including genetic predisposition, insulin resistance, lipotoxicity and dysbiosis, play a role in NAFLD pathogenesis. ${ }^{(1,2,6)}$ At the same time, the fat accumulation in the liver leads to permanent hepatocytes lesion, inflammation and fibrosis. ${ }^{(4)}$ It is believed that the main pathogenetic factors of hepatocellular lesion are elevated cytokine levels and OS due to LPO. ${ }^{(7-9)}$ Increased cytokine levels can induce hepatocyte apoptosis (TNF- $\alpha$ ), neutrophil chemotaxis (IL-8) and stellate cell activation (TNF- $\alpha$, TGF- $\beta$ ). In turn, end products of LPO can covalently bind to hepatic proteins and act as potent agents of neutrophil chemotaxis and proinflammatory cytokine stimulation. ${ }^{(10)}$ Malonic dialdehyde also activates stellate cells for collagen production, which invariably leads to cell fibrosis. ${ }^{(11)}$ In this connection, it seems appropriate to study the intensity of OS, as well as to prescribe a complex of antioxidants, selected individually, taking into account the nature of the imbalance detected, for this category of patients. ${ }^{(12,13)}$ To develop a personalized method of treatment, it is also important to take into account the patient's ethnicity. ${ }^{(14-17)}$ The purpose of this study was to assess the LPO and AOD indicators in different ethnic groups of adolescent girls with ECO and NAFLD.

\section{Materials and Methods}

A total of 20 Mongoloid teenage girls (mean age $15.00 \pm 1.73$ years) and 20 Caucasian teenage girls (mean age $16.01 \pm 2.21$ years) with NAFLD (Clinical group 1 and Clinical group 2, respectively) on the background of ECO of the first degree were examined. For comparison, data of 48 healthy Mongoloid teenage girls (mean age 15.51 1.04 years) (Control group 1) and 20 Caucasian teenage girls (mean age $15.38 \pm 1.09$ years) (Control group 2) were used. All patients underwent inpatient treatment in Children's Republican Clinical Hospital of the Ministry of Health of the Republic of Buryatia (Ulan-Ude).

Inclusion criteria for the constitutional obesity group: ECO, excess body weight of more than 95 percentiles for a given height, age and sex; absence of acute, or exacerbation of chronic diseases at the time of inclusion in the study and at least one month before it. Exclusion criteria: symptomatic and genetic forms of obesity; intake of medications that could affect body weight and estimated metabolic parameters. The criteria for the diagnosis of NAFLD: diffuse changes of liver according to ultrasound and computed tomography of the abdominal cavity, absence of cytolysis (normal levels of ALT and AST), and exclusion of infectious etiology of hepatitis.

All teenagers were subjected to general clinical examination, including anamnestic data collection, physical examination, anthropometric data analysis (BW, height, BMI), blood pressure measurement, nutritional status assessment and determination of concentration of total cholesterol and triglycerides in blood serum, glucose tolerance testing.

All clinical groups were comparable on the basic anthropometric characteristics.

The study was conducted in accordance with ethical principles of the WMA Declaration of Helsinki (1964, ed. 2013) and approved by the Ethics Committee of Scientific Centre for Family Health and Human Reproduction Problems. Written informed consent was obtained from the patient/ parent/guardian/ relative of each patient.

Blood samples $(5 \mathrm{ml})$ were collected from the ulnar vein in standard vacuum tubes with EDTA in the morning after night fasting. The intensity of LPO-AOD processes was estimated by plasma concentrations of antioxidant parameters (TAA, SOD activity, $\alpha$-tocopherol and retinol) and primary/secondary products of LPO (CDs, KD-CT, and TBARS). ${ }^{(18,19)}$ Blood plasma TAA level was detected photometrically. ${ }^{(20)}$ TBARs levels, SOD activity in hemolysate and $\alpha$-tocopherol and retinol levels in plasma were detected by fluorometry. (21)

The content of GSH and GSSG was determined by P.Y. Hissin, R. Hilf (1976), ${ }^{(22)}$ the SOD activity in the erythrocytes was determined by H.P. Misra and I. Fridovich. ${ }^{(23)}$

The measurements were conducted with a Shimadzu RF-1501 spectrophotometer (Japan) consisting of two blocks: a UV-1650PC spectrophotometer and a RF-1501 spectrofluorimeter.

The level of G-S-T in the blood plasma was detected using the ELx808 ${ }^{\mathrm{TM}}$ Absorbance Microplate Reader (BioTek Instruments, USA) and ELISA kits from Immundiagnostik AG (Germany). The measurements of the level of GR in the blood plasma and activity of GPO in the erythrocytes were conducted with a BTS-330 fully automatic Biochemistry Analyzer (Poland) using a commercial spectrophotometric assay kit (Randox Laboratories Ltd., UK).

Statistical analysis was performed using the Statistica 6.1 software package (Stat-Soft Inc., USA). Differences between groups were tested by the Mann-Whitney U-test. Group comparisons with respect to categorical variables are performed using chi-square tests. A probability value of $P<0.05$ was considered statistically significant.

\section{Results and Discussion}

Evaluation of the activity of LPO reactions in Clinical group 1 indicated an increase in the content of $\mathrm{CD}(P<0.0001)$, KD-CT $(P<0.0001)$, and TBARS $(P=0.0373)$ relative to Control group 1. In Clinical group 2, we found an increased $\mathrm{CD}$ content $(P=0.0463)$ relative to Control group 2. Interethnic differences resulted in elevated levels of KD-CT $(P=0.0176)$ in Clinical group 1 compared to Clinical group 2 (Fig.1).

In the parameters of the AOD system, we observed reduced levels of TAA $(P=0.0049)$, retinol $(P<0.0001)$ and G-S-T activity $(P=0.0383)$ in Clinical group 2 compared to Control group 2. In 
Clinical group 1, we observed reduced levels of $\alpha$-tocopherol $(P=0.0020)$, retinol $(P=0.0187)$ and G-S-T activity $(P=0.0099)$ compared to Control group 1 . There were no differences in the parameters of the AOD system between Clinical group 1 and Clinical group 2 (Figures 2 and 3).

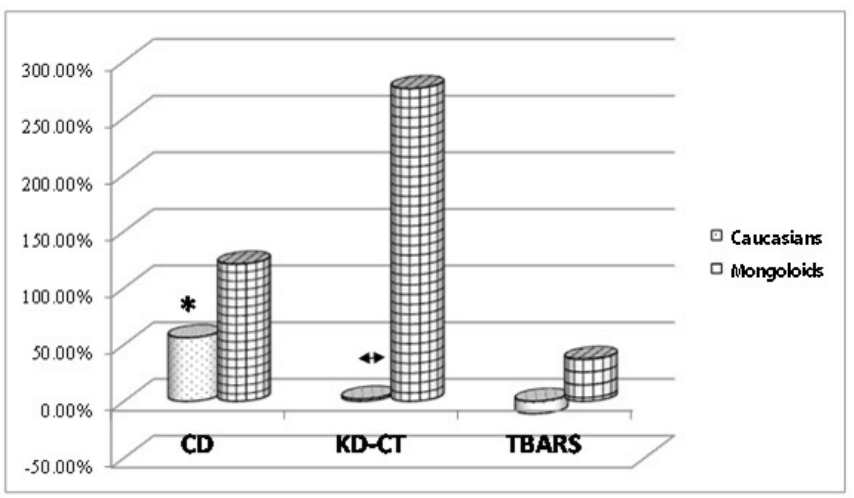

Fig. 1. Changes in LPO products in girls of different ethnic groups with ECO and NAFLD.

* - statistically significant differences compared to Control groups; $\rightarrow$-statistically significant differences between the two Clinical groups

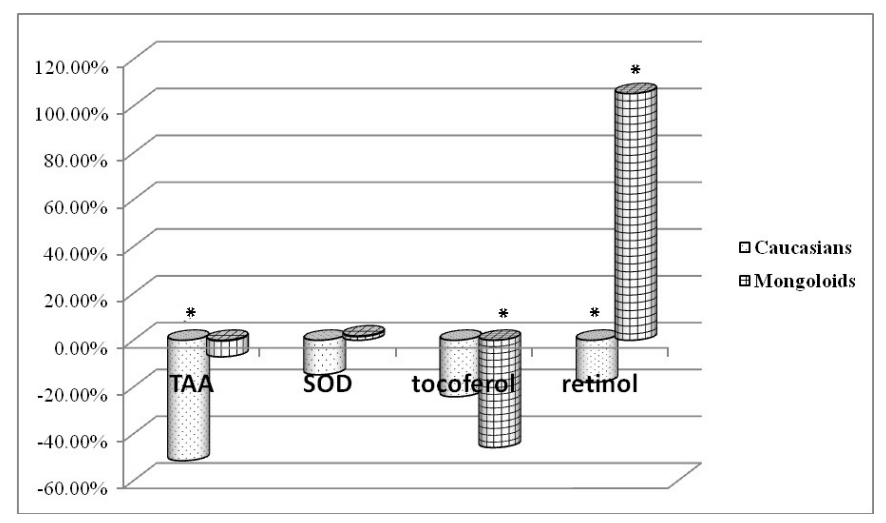

Fig. 2. Changes in the components of $A O D$ in girls of different ethnic groups with ECO and NAFLD.

* - statistically significant differences compared to Control groups

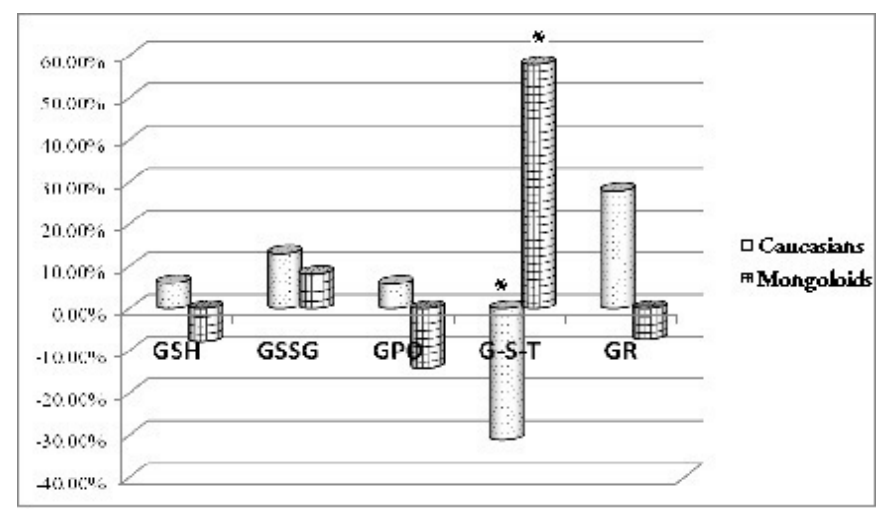

Fig. 3. Changes in glutathione system parameters in girls of different ethnic groups with ECO and NAFLD.

* - statistically significant differences compared to Control groups
We recorded an intensive accumulation of the primary, secondary and end products of LPO in Mongoloid teenage girls with ECO and NAFLD. These changes are likely to be associated with metabolic changes in the liver that occur as a result of obesity. In such cases, there is an excessive accumulation of lipids (including fat deposits in hepatocytes), which enhances LPO processes, and an increase in the amount of free fatty acids, triglycerides in the blood, etc. ${ }^{(24,25)}$ The inflammatory process is activated with the hyperproduction of proinflammatory cytokines. ${ }^{(26)}$ The subsequent development of the process promotes fibrogenesis in liver cells. ${ }^{(10,11)}$ Excessive accumulation of toxic LPO products, especially expressed in Mongoloid patients, may exacerbate the initial morphofunctional changes in cells, and, accordingly, aggravate the course of the disease.

It is known that the parameters of the AOD system are a significant diagnostic criterion of adaptation reactions of the body. ${ }^{(28-30)}$ We observed a decrease in the values of TAA (as an integral parameter of the AOD system), retinol and glutathione transferase activity in Caucasian girls with obesity and NAFLD. Similar phenomena were found in Mongoloids with severe $\alpha$-tocopherol deficiency. In a study by M. Guerendiain et al., an increase in plasma levels of lipid-corrected $\alpha$-tocopherol $(P=0.001)$ was associated with reduced adiposity and greater weight loss in overweight and

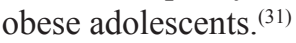

It is known that $\alpha$-tocopherol and retinol are the most important antioxidants and are essential substances. ${ }^{(28,31)}$ $\alpha$-tocopherol is an important chain-breaking antioxidant that prevents free radical reactions and LPO. ${ }^{(32,33)} \alpha$-tocopherol has a high membrane protective and antimutagenic activity, whereby interacting with natural antioxidants of other classes it acts as an essential regulator of oxidative homeostasis of cells and tissues. ${ }^{(34,22)}$ The antioxidant function of retinol is expressed in the protection of biomembranes from damage by active forms of oxygen. ${ }^{(35)}$

It is known that the liver is the main pool of fatand water-soluble vitamins; therefore, we confirmed in our study the decrease in the content of these vitamins in NAFLD and their corresponding deficiency at the system level in the adolescents of both ethnic groups. In our study, a more severe deficiency of AOD factors was detected in Mongoloid girls, reflected by intense accumulation of LPO products. Similar results were also observed in China. ${ }^{(36)}$ Our data may be associated with eating disorders of indigenous ethnic representatives, and a corresponding increase in obesity among them, which is confirmed by our previous studies. ${ }^{(14,29)}$

\section{Conclusion}

Thus, the results obtained indicate high activity of LPO processes and severe deficiency of antioxidant factors in Mongoloid girls with ECO and NAFLD in comparison with Caucasian girls with obesity and NAFLD and the control group. The obtained results allow us to recommend administration of antioxidant drugs in addition to basic therapy in comprehensive treatment of patients with NAFLD. 


\section{Competing Interests} interests.

The authors declare that they have no competing

\section{References}

1. Marion AW, Baker AJ, Dhawan A. Fatty liver disease in children. Arch Dis Child. 2004;89(7):648-652.

2. Angulo P. Obesity and nonalcoholic fatty liver disease. Nutr Rev. 2007;65(6 Pt 2): S57-63.

3. Sheth SG, Chopra S. Epidemiology, clinical features, and diagnosis of nonalcoholic fatty liver disease in adults. Waltham (MA): UpToDate; 2017.

4. McCullough AJ. Pathophysiology of nonalcoholic steatohepatitis. J Clin Gastroenterol. 2006;40Suppl 1:S17-29. doi: 10.1097/01.mcg.0000168645.86658.22.

5. Guijarro de Armas MG, Monereo Megías S, Navea Aguilera C, Merino Viveros M, Vega Piñero MB. [Nonalcoholic fatty liver in children and adolescents with excess weight and obesity]. Med Clin (Barc). 2015 Jan 20;144(2):558. doi: 10.1016/j.medcli.2014.02.018. [Article in Spanish]. 6. Rinella ME. Nonalcoholic fatty liver disease: a systematic review. JAMA. 2015 313(22):2263-73. doi: 10.1001/ jama.2015.5370.

7. Darenskaya MA, Gavrilova OA, Rychkova LV, Zhdanova LA, Buldaeva EA, Grebenkina LA, et al. Characteristics of lipid peroxidation processes and antioxidant status in teenagers-boys of different ethnic groups with exogenous constitutional obesity and non-alcoholic fatty liver disease. International Journal of Biomedicine. 2018;8(4):306-310. doi: 10.21103/Article8(4)_OA7

8. Darenskaya MA $\bar{A}$, Rychkova LV, Gavrilova OA, Zhdanova VL, Bimbaev AB-Zh, Grebenkina LA, et al. Lipid peroxidation parameters in mongoloid-patients with obesity and hepatosis. Free Radical Biology \& Medicine. 2018;120(S1):S61. doi: 10.1016/j.freeradbiomed.2018.04.201 9. Darenskaya MA, Grebenkina LA, Sholokhov LF, Rashidova MA, Semenova NV, Kolesnikov SI, Kolesnikova LI. Lipid Peroxidation Activity in Women with Chronic Viral Hepatitis. Free Radical Biol. Med. 2016;100(Suppl):S192.

10. Mehta K., Van Thiel D.H., Shah N, Mobarhan S.. Nonalcoholic fatty liver disease: pathogenesis and the role of antioxidants. Nutr Rev. 2002;60(9):289-93 .

11. Köroğlu E, Canbakan B, Atay K, Hatemi İ, Tuncer M, Dobrucalı A, Sonsuz A, Gültepe I, Şentürk H. Role of oxidative stress and insulin resistance in disease severity of non-alcoholic fatty liver disease. Turk J Gastroenterol. 2016;27(4):361-6. doi: 10.5152/tjg.2016.16106.

12. Lirussi F, Azzalini L, Orando S, Orlando R, Angelico F. Antioxidant supplements for non-alcoholic fatty liver disease and/or steatohepatitis. Cochrane Database Syst Rev. 2007 Jan 24;(1):CD004996.

13. Darenskaya MA, Rychkova LV, Kolesnikov SI, et al. Oxidative stress parameters in adolescent boys with exogenous-constitutional obesity. Free Radical Biology \& Medicine. 2017;112:129-30.

*Corresponding author: Marina A. Darenskaya, PhD, ScD. . Scientific Centre for Family Health and Human Reproduction Problems, Irkutsk, the Russian Federation. E-mail: marina darenskaya@inbox.ru
14. Kolesnikova LI, Darenskaya MA, Grebenkina LA, Suturina LV, Labygina AV, Semenova NV, et al. [Characteristics of the antioxidant system of healthy people of main ethnic groups living near Baikal Lake]. Vopr Pitan. 2012;81(3):4651..[Article in Russian].

15. Kolesnikova LI, Darenskaya MA, Grebenkina LA, Dolgikh MI, Astakhova TA, Semenova NV. [Gender differences in parameters of lipid metabolism and of level of antioxidants in groups of juveniles--the Evenks and the Europeans]. Zh Evol Biokhim Fiziol. 2014 Jan-Feb;50(1):31-7.[Article in Russian]. 16. Kolesnikova LI, Darenskaya MA, Grebenkina LA, Labygina AV, Suturina LV, Dolgikh MI, et al. Activity of lipid peroxidation in infertile women from different populations. Bull Exp Biol Med. 2012;154(2):203-5.

17. Kolesnikova LI, Kolesnikov SI, Darenskaya MA, Grebenkina LA, Semenova NV, Osipova EV, et al. Lipid Status and Predisposing Genes in Patients with Diabetes Mellitus Type 1 from Various Ethnic Groups. Bull Exp Biol Med. 2015;160(2):278-80. doi: 10.1007/s10517-015-3149-5.

18. Volchegorskiĩ IA, Nalimov AG, Iarovinskiı̌ BG, Lifshits RI. [Comparison of various approaches to the determination of the products of lipid peroxidation in heptane-isopropanol extracts of blood]. Vopr Med Khim. 1989;35(1):127-31. [Article in Russian].

19. Gavrilov VB, Gavrilova AR, Mazhul' LM. [Methods of determining lipid peroxidation products in the serum using a thiobarbituric acid test].Vopr Med Khim. 1987;33(1):118-22. [Article in Russian].

20. Klebanov GI, Babenkova IV, Teselkin IuO, Komarov OS, Vladimirov IuA. [Evaluation of the antioxidative activity of blood plasma using yolk lipoproteins]. Lab Delo. 1988;(5):5962.[Article in Russian].

21. Cherniauskene RCh, Varshkiavichene ZZ, Gribauskas PS. [Simultaneous fluorometric determination of the concentrations of vitamins E and A in blood serum]. Lab Delo. 1984;(6):362-5.[Article in Russian]

22. Hisin PJ, Hilf R. A fluorometric method for determination of oxidized and reduced glutathione in tissues. Anal Biochem. 1976;74(1):214-26.

23. Misra HP, Fridovich I. The role of superoxide anion in the autoxidation of epinephrine and a simple assay for superoxide dismutase. J Biol Chem. 1972;247(10):3170-5.

24. Heymsfield SB, Wadden TA. Mechanisms, Pathophysiology, and Management of Obesity. N Engl J Med. 2017;376(3):254-266. doi: 10.1056/NEJMra1514009.

25. Camacho S, Ruppel A. Is the calorie concept a real solution to the obesity epidemic? Glob Health Action. 2017;10(1):1289650. doi: 10.1080/16549716.2017.1289650.

26. Kolosov YuA, Kolesnikov SI, Anischenko AP, Burdukova EV, Gurevich KG. [Overweight and obesity in children, adolescents and adults: causes of development and risk factors]. Patogenez [Pathogenesis].2016; Vol. 14 (4): 9-14. [Article in Russian].

27. Sun M, Huang X, Yan Y, Chen J, Wang Z, Xie M, Li J. Rac1 is a possible link between obesity and oxidative stress in Chinese overweight adolescents. Obesity (Silver Spring). 2012;20(11):2233-40. doi: 10.1038/oby.2012.63.

28. Kolesnikova LI, Semyonova NV, Grebenkina LA, Darenskaya MA, Suturina LV, Gnusina S. Integral indicator of oxidative stress in human blood. Bull Exp Biol Med. 2014;157(6):715-7. doi: 10.1007/s10517-014-2649-z.

29. Darenskaya MA, Gavrilova OA, Rychkova LV, Kravtsova OV, Grebenkina LA, Osipova EV, et al. The 
assessment of oxidative stress intensity in adolescents with obesity by the integral index. International Journal of Biomedicine.2018;8(1)37-41. doi: 10.21103/Article8(1)_OA5 30. Kolesnikova LI, Darenskaya MA, Grebenkina LA, Dolgikh MI, Semenova NV. [Adaptive reactions of lipid metabolism in native and alien female representatives of Tofalaria population living under extreme environmental conditions]. Zh Evol Biokhim Fiziol. 2014;50(5):343-8. [Article in Russian].

31. Guerendiain M. Mayneris-Perxachs J, Montes R, López-Belmonte G, Martín-Matillas, M, Castellote AI. Relation between plasma antioxidant vitamin levels, adiposity and cardio-metabolic profile in adolescents: Effects of a multidisciplinary obesity programme. Clinical nutrition. 2017:36(1):209-217.

32. Meydani M. Vitamin E. Lancet.1995;345(8943):170-5.

33. Kaul N, Devaraj S, Jialal I. Alpha-tocopherol and atherosclerosis. Exp Biol Med (Maywood). 2001;226(1):5-12. 34. Pisoschi AM, Pop A. The role of antioxidants in the chemistry of oxidative stress: A review. Eur J Med Chem. 2015; 97:55-74. doi: 10.1016/j.ejmech.2015.04.040.

35. Kodentsova VM, Vrzhesinskaia OA, Mazo VK. [Vitamins and oxidative stress]. Vopr Pitan. 2013;82(3):11-8. [Article in Russian]

36. Karbownik-Lewinska M, Szosland J, Kokoszko-Bilska A, Stępniak J, Zasada K, Gesing A, Lewinski A. Direct contribution of obesity to oxidative damage to macromolecules. Neuro Endocrinol Lett. 2012;33(4):453-61. 